\title{
PULSED OPERATION OF THE CERN SPS COLLIDER
}

\author{
R. Lauckner \\ CERN - 1211 Geneva 23, Switzerland \\ 1211, Geneva 23
}

\section{Summary}

The SPS is limited to $315 \mathrm{GEV}$ per beam when running as a proton antiproton collider. This is due to ohmic heating of the coils of the main magnets. The limit can be overcome by pulsing the magnets between $100 \mathrm{GeV}$ and $450 \mathrm{GeV}$. During a recent physics run to study p pbar collisions at 900 GeV c.m. energy, beams of protons and antiprotons were collided under these pulsed conditions. The development of this facility and the resulting machine performance will be described.

\section{Introduction}

In 1982 the UA5 collaboration proposed 1 an exploratory experiment to observe p-pbar collisions at $900 \mathrm{GeV} \mathrm{c} . \mathrm{m}$. energy, The UA5 detector, a streamer chamber, had already observed hadron collisions at $53 \mathrm{GeV} c \cdot m$. in the ISR and $540 \mathrm{GeV}$ c.m. in the SPS. By colliding beams of protons and antiprotons whilst ramping the SPS between $100 \mathrm{GeV}$ and $450 \mathrm{GeV}$ an overview of hadron physics in a very large range of energies could be otained.

During the later half of 1983 and 1984 hardware and software modifications were undertaken and led to the successful storage of 2 proton bunches during machine development sessions in the summer of 1984 . These bunches had an intensity of $\sim 3 \times 10^{10}$ and an intensity lifetime of up to 4 hours. Encouraged by this result a physics run was scheculed for March 1985. The first p-pbar collisions at $900 \mathrm{GeV} \mathrm{c.m.} \mathrm{were} \mathrm{observed} \mathrm{on}$ March 16th. Data taking by UAS started the next day and beams were collided whilst ramping between $100 \mathrm{GeV}$ and $450 \mathrm{GeV}$ for a total of 95 hours before the end of the run on April 4th. Initial luminosities were about $2 \times 10^{26} \mathrm{~cm}^{-2} \mathrm{~s}^{-1}$ at $450 \mathrm{GeV}$ and over 500 000 events were recorded by UA5 and UAI (who ran as a second user).

\section{Machine cycle}

Figure 1 shows the machine energy cycle used to store the beams.

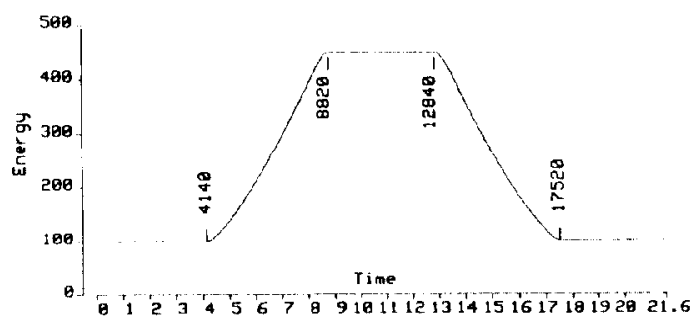

Figure 1: The Storage Cycle

Various limits had to be respected by this cycle. It had to remain within the maximum r.m.s. power limits imposed by heating of the magnet coils, the high energy plateau was limited in length to avoid overheating the thyristors in the rectifier stations and the ramps were designed taking into account the available $r f$ power and magnet power supply voltages. Within these limits the most Favourable duty cycle for studying p-pbar collisions was chosen.
The result provides a $19 \%$ duty cycle at $450 \mathrm{GeV}$ and $38 \%$ at $100 \mathrm{GeV}$, the lower energy was chosen bearing in mind the requirement to remain fairly close to ISR energies. Background conditions were clean enough to allow data taking on the ramps as well as the plateaux.

However, a second machine energy cycle was required to inject the CPS beams at $26 \mathrm{GeV}$ and accelerate them to $100 \mathrm{GeV}$. It was decided that to achieve this novel mode of SPS operation two separate machine cycles would be used which could be combined in any sequence to form a supercycle. Figure 2 shows the injection cycle. This cycle was designed with the following features.

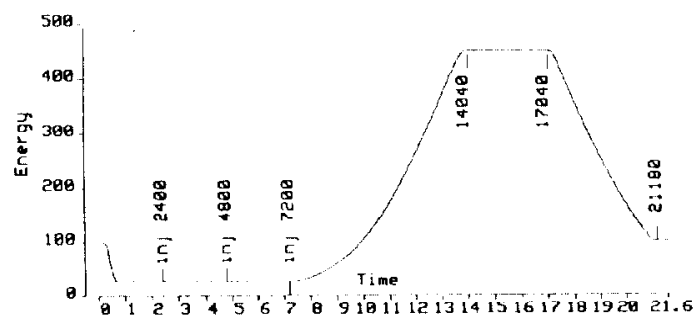

Figure 2: The Injection Cycle

The end points were at the same energy as those of the storage cycle, this allows interleaving with the storage cycle. Two proton bunches and one antiproton bunch could be injected from the CPS (repetition time 2.45 ). Ramp rates were somewhat faster that the storage cycle, longitudinal beam lifetime was not important so the high energy plateau was made as long as possible to bring the power consumption of the bending magnets up to $28 \mathrm{MW}$ against $35 \mathrm{MW}$ of the storage cycle to avold changes in magnet temperatures. This last property was a reason for pulsing to $450 \mathrm{GeV}$ in the injection cycle. A second reason was the need to make succeeding storage cycles magnetically the same. This would not have been the case if the initial ramp-up of the storage cycle were to follow a $26 \mathrm{GeV}$ plateau if preceeded by an injection cycle or a $450 \mathrm{GeV}$ plateau if preceeded by a storage cycle.

\section{Supercycling the SPS}

Some hardware developments but chiefly software effort was required to run the SPS as a pulsed collider. These activities were divided into two areas, tuning a pulsing machine to a precision comparable with that of a storage ring and converting a single cycle machine into a simple supercycling machine.

In order to set-up, tune and coast beams several combinations of the injection cycle $I$ and the storage cycle $S$ were of interest.

$\begin{array}{ll}\text { (1) } & \text { I, I, . . } \\ \text { (2) } & \text { I, S, I, S, . . } \\ \text { (4) I, S, S, I, S, S, . } & \text { s, s, .. }\end{array}$


A new central timing pulse generator was built which could supply the timing pulses required to control the machine components for any combination of the 2 cycles. The software of the dedicated NORD 100 computer which generates the reference signal for the main dipole and quadrupole power supplies was extended to switch between the two magnetic cycles as required. offline console software for the pre-generation of these references was also extended.

Several function generators which control pulsing components of the sps were equipped with second memory cards, extended low level interface software and console software.

A high priority driver was implemented in one of the SPS computers which interpreted the operators request of the supercycle pattern, sequenced the timing pulse generator, and directed the remaining real time tasks required to achieve supercycling. This program was surprisingly simple $(\sim 1$ weok to write).

Additionally high level software used for beam diagnostics had to be modified to allow the operator to direct his attention to the relevant cycle.

Reasonably user-friendly software was provided for controlling the principle accelerator components and measuring the principle beam parameters in this environment. Because of the exploratory nature of the experiment no low beta insertions or pulsed closed orbit correction could be provided.

\section{Tuning the SPS}

When running as a d.c. collider 2 it takes about 8 hours to set-up and tune the pulsed functions of the machine to accelerate beams from $26 \mathrm{GeV}$ to $315 \mathrm{GeV}$. Typically the tune is adjusted to an accuracy of \pm .01 . In d.c. storage the working point is adjusted to an eccuracy of $t .001$ and the current control loops of the main power supplies keep the tune constant to $\sim \pm .0001$. For the pulsed collider, hardware and software techniques 3 were developed with the aim of adjusting the tune to a precision better than +.005 during the continuous ramping. Two complementary methods were provided for measuring the tune. The first consisted of kicking the beam every $60 \mathrm{~ms}$ throughout the cycle and performing a fast Fourier transform on the position data from 256 turns following the kick. This method is robust, working well over a wide range of beam conditions and has an accuracy of \pm .005 . The second method was to excite the beam at a multiple of the betatron frequency and feed the response back to the excitation kicker via a phase lock loop. This has an accuracy of better than \pm .001 . Both results could be fed back into the power supply reference via a software loop. These techniques could also be extended to a measurement of the chromaticity throughout the cycle. The machine development sessions were mainly devoted to the testing of these techniques and they were a vital factor in the success of the experiment.

\section{Performance for physics}

Table I shows the performance of the pulsed collider for a typical good run. These figures although some orders of magnitude below the achievements in d.c, collider mode were nevertheless well suited to the experiment. The original proposal called for a few tens of hours beam time with luminosities $\gtrsim 10^{25} \mathrm{~cm}^{-2} \mathrm{~s}^{-1}$.

Table I

\section{Pulsed collider typical good run}

AA stacking rate

Time between $f$ ills

stacking time between fills

Transfer efficiency

Initial pbar intensity

Initial p+ intensity

Normalized emittances $p+$

Normalized emittances $\bar{p}$

Beam beam parameter $\xi$

$\mathrm{B}_{\mathrm{H}, \mathrm{V}}$

Initial luminosity ( $450 \mathrm{GeV})$

Luminosity lifetime

Bunch 1 ifetimes

Duration of coast

Table II shows the average performance and the production over the period. Running with a minimum biased trigger the experiments UAS and UA1 were both limited by their data taking rates at $450 \mathrm{GeV}$ with the Iuminosities achieved. Background rates were generally low enough for the experiments to take data over the whole cycle, apart from two discrete points near each end of the down ramp. Rates were observed integrated over the whole cycle, 1 second and $10 \mathrm{~ms}$. The two points were only visible on the fast signal.

\section{Table II}

\section{Pulsed collider total production}

Number of runs for physics

Average duration of run

Average luminosity lifetime

Average initial luminosity at $\bar{\gamma}$

Average yield at $\bar{y}$

Total yield at $\bar{y}$

Total yield at $450 \mathrm{GeV}$

$\begin{array}{ll}37 & \\ 2.5 & \mathrm{hrs} \\ 2 & \mathrm{hrs} \\ 1 \times 10^{26} \mathrm{~cm}^{-2} \mathrm{~s}^{-1} \\ 0.5 & \mu \mathrm{b}^{-1} \\ 19 & \mu \mathrm{b}^{-1} \\ 6.9 & \mu \mathrm{b}^{-1}\end{array}$

Bean parameters

Tune

Figure 3 shows the tune diagram at $100 \mathrm{GeV}$ with the initial conditions of Table $I$ and $a$ nominal working point of $\mathrm{QH}_{\mathrm{H}} \mathrm{V}=26.688,26.678$

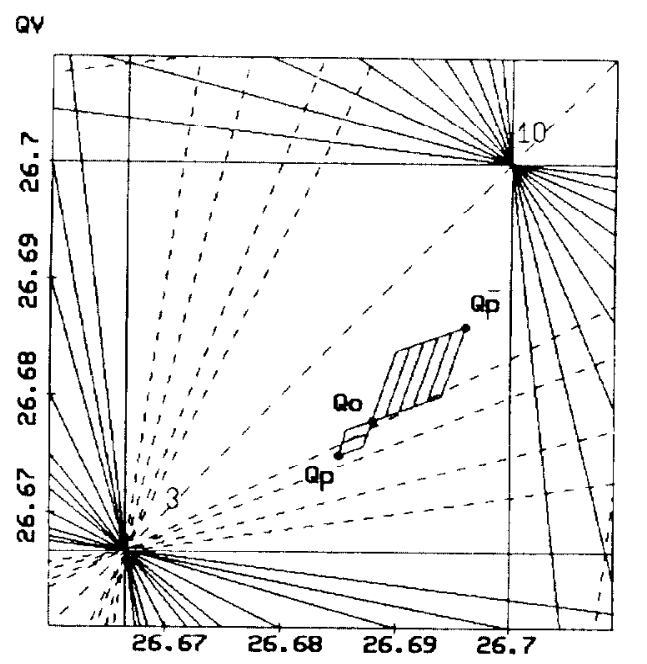

Figure 3: Tune diagram at $100 \mathrm{GeV}$

There are margins of about .005 between the antiprotons and the $10^{\text {th }}$ order beam-beam driven resonance and between the protons and the stopband of the third order resonance. 
Figure 4 shows a frequency distribution of the vertical tune measured every $6 \mathrm{~ms}$ through the $21.6 \mathrm{~s}$ cycle after correction. This tune was measured at low intensity $\sim 2 \times 10^{10}$ /bunch to avoid coherent tune shifts which are $\sim .005$ at the operating proton intensity, The horizontal tune adjustment was of similar precision.

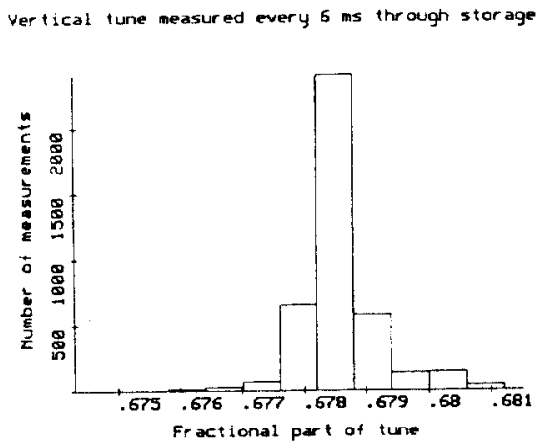

Figure 4: Precision of the tune correction

variation of the tune during a coast was checked by observing the natural schottky noise and was $<.002$.

\section{Chromaticity}

This was measured every 60 ms throughout the cycle and was adjusted such that $0<\xi_{H, V}<0.25$. Again this was an adequate adjustment.

\section{Proton intensity}

The energy ramps limited the longitudinal acceptance to $0.5 \mathrm{eV} . \mathrm{s}$. The micro-wave instability has a threshold of $\sim 9 \times 10^{10}$ at injection. Consequently the injected intensity was kept at $\sim 8 \times 10^{10}$ which after scraping in the first few minutes settled to an initial value of $6 \times 10^{10}$ bunch.

\section{Antiproton intensity}

This parameter never exceeded $10^{10 / b u n c h}$ due to the AA stacking rate and the low luminosity lifetime. Poor transfer efficiencies compared to normal collider operation reflected the heavy demands of setting-up the storage cycle.

\section{Intensity lifetimes}

Both proton and antiproton bunches had intensity lifetimes which climbed from about 1 hour to 6-7 hours in the first hour of coast. In an experiment with no antiprotons in the machine a proton bunch of large transverse emittance was collimated by reducing the r.f. voltage for a few cycles. This produced a bunch of $3 \times 10^{20}$ protons with a normalised transverse emittance of $35 \pi \times 10^{-6}$ rad.m. The length of the bunch measured at 1 sigma was reduced from $0.6 \mathrm{~ns}$ to 0.5 ns at $100 \mathrm{GeV}$ by the collimation. The intensity lifetime rose to 50 hours then fell slowly to about 6 hours after 1 hours. Ramping of the beams leads to generation of r.F. noise which dominates the intensily lifetimes of both beams.

\section{Emittances}

The protom emittances were comparable with those achieved during d.c. collider runs although at lower intensity. The larger antiproton emittance reflects again the poor transfer efficiency but as the antiprotons were kept clear of the $10^{\text {th }}$ order resonances, this did not lead to any loss of particles. Emittance lifetime was of the order of one day.

Luminosity lifetime

Intensity lifetimes of a few hours were dominant in determining the luminosity lifetime, the transverse emittances being essentially constant during a coast.

\section{RF lifetimes}

The r.f. beam control system used was the same system as is used in d.c. collider ${ }^{4}$. As in that mode the bunches are latently unstable and indeed some early physics coasts suffered occasional losses from one or other of the proton bunches. This was quickly resolved by reducing the r.f. voltage at 450 $\mathrm{GeV}$ from $4 \mathrm{MV}$ to $2 \mathrm{MV}$ and adjusting the quadrupole dumping loops.

In the collider the r.f. noise originates from the electronics of the beam control system and has been steadily reduced until the r.f. bunch lifetime is now $>100$ hours. In pulsed collider noise arises from the continual ramping. One such source was identified during the 1984 machine development sessions and originated from the discrete nature of the input to the stable phase program which is generated from a reference bending magnet and produces a stepped input every 0.1 gauss. This noise source was replaced by a smoothed static reference signal and perhaps acounted for the asymtotic intensity lifetime increasing from 4 to 7 hours.

A second source of noise, which remains, arose from the change of the stable phase, which is an imposed noise source, whose average spectral density at the synchrotron frequency determines its contribution to the lifetime. Fourier analysis of the ideal stable phase variation shows that the amplitude of this noise is determined by the length of the transitions between the ramps and the plateaux and by the peak stable phase. Calculation of the intensity lifetime from the measured noise levels gives a lifetime of 4.7 hours.

\section{Acknowledgements}

A large number of specialists made essential contributions to this project. The final success owes much to co-operation, enthusiasm and persistence.

\section{References}

11 An Exploratory Investigation of ppbar Interactions at $800900 \mathrm{GeV}$ e.m. Energy at the SPS Collider - IJA5 Collaboration CERN/SPSC 82-75 SPSC/P 184 - 15 October 1982

2] The CERN SPS Proton-Antiproton Collider B. de Raad This Conference

3) Tune Measurement and Control at the CERN-SPS $R$. Bossart et al This Conference

4) RF Noise Performance of the CERN Proton Antiproton Collider D. Boussard and T.P.R. Linnecar $12^{\text {th }}$ Int. Conf. on High Energy Accelerators 11-16 August 1983 